\title{
Épuration des polluants issus de la combustion domestique du bois
}

\author{
L. Robert ${ }^{1}$ \\ 1 CSTB, 11 rue Henri Picherit, BP 82341, 44323 Nantes Cedex 03 - France \\ e-mail: robert@cstb.fr
}

\begin{abstract}
Résumé - Cet article présente la démarche développée par le CSTB pour tenter de réduire la pollution issue de la combustion domestique du bois. Il s'agit de développer des procédés épurateurs pour éliminer partiellement les composés polluants ; procédés ayant l'originalité d'être partie intégrante du conduit de fumées. Afin de choisir les différentes voies d'épuration à considérer, une étude préliminaire sur la caractérisation des fumées produites par le fonctionnement d'un insert à bois a été réalisée. Les poussières à raison de 2 à $20{\mathrm{~g} \cdot \mathrm{kg}^{-1}}^{-1}$ de bois sec brûlé, le monoxyde de carbone (CO) et les hydrocarbures totaux (HC) à raison respectivement de 70 à 280 et 2 à $9 \mathrm{~g} \cdot \mathrm{kg}^{-1}$ de bois sec brûlé, sont visés. A ce jour deux techniques sont développées. La séparation des poussières utilise l'effet centrifuge d'une pâle motorisée couplée à un jeu d'ailettes. Sur banc de simulation, $56 \%$ des poussières sont retenues par le prototype. Pour ce qui est des imbrûlés gazeux $\mathrm{CO}$ et $\mathrm{HC}$, l'emploi d'un catalyseur au platine a été développé. Mis en situation réelle, des rendements moyens du catalyseur de $73 \%$ pour le $\mathrm{CO}$ et de $37 \%$ pour les $\mathrm{HC}$ sont obtenus.
\end{abstract}

\begin{abstract}
Reduction of Pollutants Resulting from Domestic Heating Using Firewood - This paper presents the method developed by the CSTB to reduce the pollution from the domestic heating using firewood. The objective is to develop processes to eliminate partially the principal pollutants. The originality of these processes is to be integrated of the flue duct. To choose the ways of treatment to be considered, a preliminary study on the characterization of smokes produced by a fireplace was realized. Dusts (rate between 2 in $20 \mathrm{~g} \cdot \mathrm{kg}^{-1}$ of burned dry wood), carbon monoxide (CO) and unburned hydrocarbons (HC) (respectively of 70 in 280 and 2 in $9 \mathrm{~g}^{\mathrm{kg}} \mathrm{g}^{-1}$ of burned dry wood), are aimed. Two processes are developed. For the dusts separation, the centrifugal effect by using a blade motorized coupled with a set of fins, is used. $56 \%$ of dusts are retained by the prototype during the tests on the simulation bench. As for the $\mathrm{CO}$ and $\mathrm{HC}$, the use of a platinum catalyst was developed. Put in real situation, an average of $73 \%$ for $\mathrm{CO}$ is eliminated and $37 \%$ for the $\mathrm{HC}$.
\end{abstract}




\section{INTRODUCTION}

Devant la nécessité de limiter les émissions de gaz à effet de serre et de promouvoir les matières premières renouvelables, et ce, dans le cadre d'un développement durable, il ne fait plus de doute que le bois constitue une alternative de choix au remplacement des combustibles fossiles. Cependant, la combustion du bois génère différents types de polluants en quantité non négligeable ; notamment en terme d'émission de monoxyde de carbone, d'hydrocarbures imbrûlés et de poussières. En France, pays où la forêt couvre la majeure partie du territoire, où la valorisation de la filière bois-énergie est en plein essor, et où la politique environnementale reste une préoccupation de première importance, nous constatons que très peu, voire quasiment pas d'études sont à même de proposer des solutions visant à réduire les émissions polluantes issues du chauffage domestique au bois. C'est dans ce contexte que des recherches sont menées au CSTB afin de proposer une nouvelle génération de conduits de fumées, qui par l'action combinée de plusieurs procédés d'épuration, permettrait de réduire de façon sensible les émissions de polluants dans l'atmosphère.

Cet article s'articulera selon deux grands axes. Seront présentés une caractérisation approfondie des polluants générés lors de la combustion du bois dans un insert, suivie des différentes voies d'épuration envisagées. Cependant, au préalable, un premier paragraphe dédié au banc d'essai ainsi qu'à l'ensemble des mesures effectuées, sera détaillé afin de mieux cerner le contexte de cette recherche.

\section{DÉMARCHE EXPÉRIMENTALE}

La démarche expérimentale suivie s'est voulue très proche de l'utilisation que fait le particulier de son foyer à bois, et ce, afin de pouvoir obtenir une quantification réaliste des émissions polluantes issues de ce type d'appareil. Il a été utilisé pour cette étude, un insert à bois muni d'une seule amenée d'air comburant. Cet appareil a été équipé de conduits de fumées de diamètre intérieur $200 \mathrm{~mm}$, en acier inox double peau sur une hauteur de $5 \mathrm{~m}$, et ce, afin de représenter la hauteur fréquemment rencontrée chez l'usager. Ce conduit a été instrumenté de façon à pouvoir analyser en continu, via une centrale d'acquisition, les évolutions de différents paramètres et polluants, figure 1 , à savoir :

- la température des fumées ;

- la vitesse des fumées ;

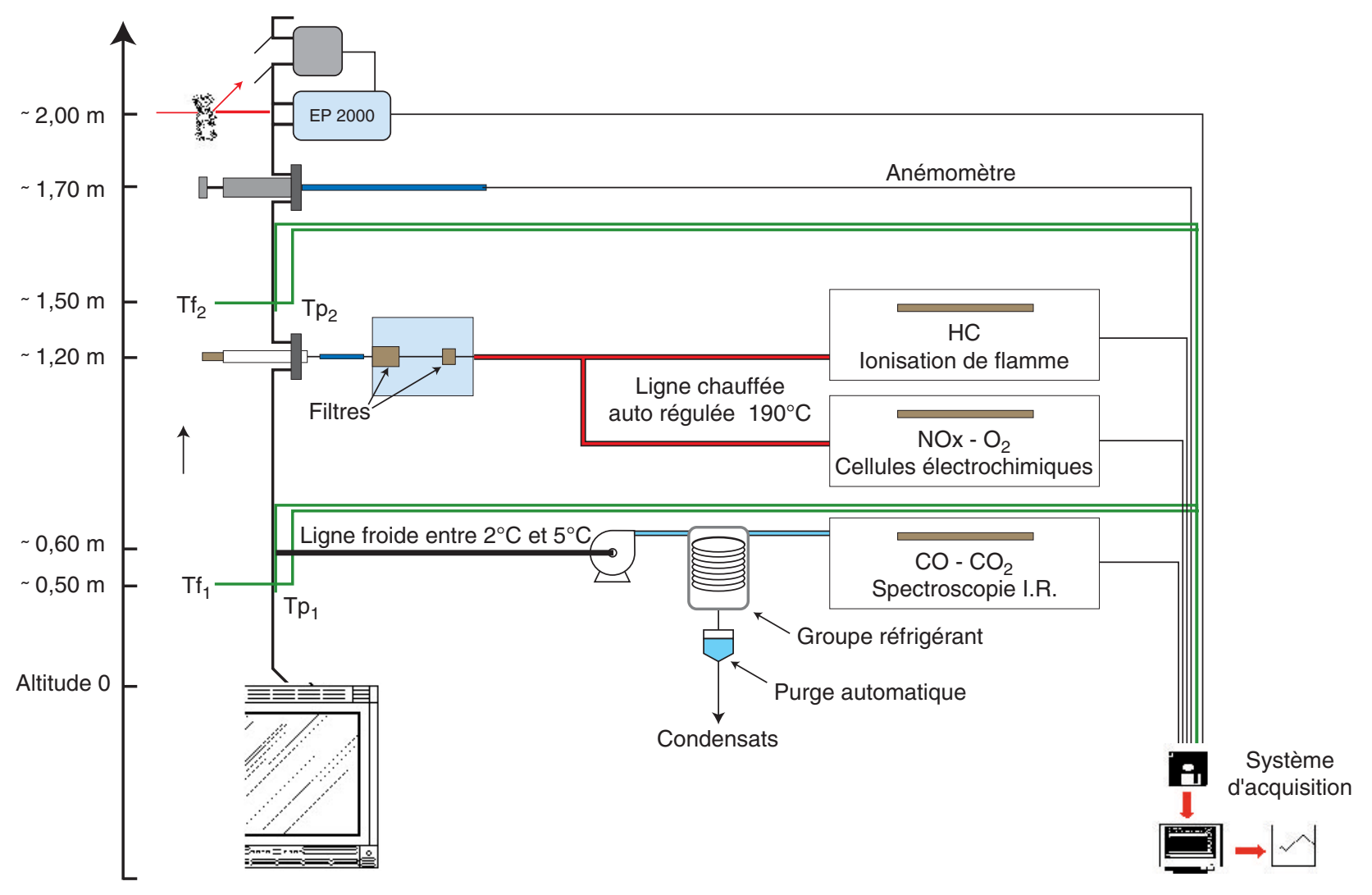

Figure 1

Montage expérimental.

Experimental bench. 
- les émissions de poussières (PM) par post diffusion laser ;

- les émissions d'hydrocarbures totaux (HC) par ionisation de flamme ;

- les émissions de monoxyde et dioxyde de carbone (respectivement $\mathrm{CO}$ et $\mathrm{CO}_{2}$ ) par spectroscopie IR ;

- les émissions d'oxydes d'azote et de soufre (respectivement $\mathrm{NO}_{\mathrm{x}}$ et $\mathrm{SO}_{\mathrm{x}}$ ) par cellules électrochimiques.

Une analyse ponctuelle des hydrocarbures aromatiques polycycliques (HAP) par HPLC, réputés pour leur toxicité, ainsi qu'une caractérisation physico-chimique des poussières ont été réalisées. Enfin, afin d'approfondir la connaissance des ces émissions polluantes, on a cherché à quantifier l'influence de certaines pratiques du consommateur, notamment le fonctionnement en allure réduite par rapport à l'allure nominale et l'emploi de bois de différentes humidités. Trois classes d'humidité ont été retenues :

- $\mathrm{C}_{1}$ : le bois très sec dont l'humidité est inférieure à $12 \%$;

$-\mathrm{C}_{2}$ : le bois sec dont l'humidité est comprise entre 15 et $20 \%$;

- $\mathrm{C}_{3}$ : le bois humide dont l'humidité dépasse $25 \%$.

Pour tous les essais, l'essence retenue a été le chêne conditionné en bûches fendues de $50 \mathrm{~cm}$. L'ensemble des résultats est exprimé en terme de facteurs d'émission, et de ce fait exprimé en $\mathrm{g}$ de polluant émis ramené au $\mathrm{kg}$ de bois sec brûlé.

\section{POLLUTION ENGENDRÉE}

\section{1 Émissions de poussières}

Le terme poussière regroupe, dans cet article, les petites particules solides, qui issues de la combustion du bois sont émises à l'atmosphère par le biais des fumées. Généralement, les particules rencontrées dans la combustion du bois, peuvent être classées en deux catégories : les cendres volantes et les poussières. Notre recherche s'est plus particulièrement orientée sur les poussières, qui beaucoup plus fines, représentent la fraction de particules solides véhiculées par les fumées jusqu'à l'atmosphère. Leur « petite » taille, facilitant leur transport et leur inhalation, font d'elles un réel enjeu environnemental et de santé publique. En outre, leur présence en tant qu'aérosol dans l'atmosphère contribue à la réduction de la visibilité, la salissure des façades ; elles peuvent également contribuer à des phénomènes tels que la corrosion et induire une gène pour la photosynthèse.

Les émissions de poussières au cours de la combustion de bois dépendent fortement de l'allure de combustion. En allure nominale, les émissions ne sont importantes que durant la phase de démarrage du feu ; les concentrations pouvant atteindre $2 \mathrm{~g} \cdot \mathrm{m}^{-3}$. Elles diminuent ensuite rapidement à des valeurs beaucoup plus faibles, de l'ordre de 40 à $50 \mathrm{mg} \cdot \mathrm{m}^{-3}$. On notera que généralement, en allure nominale, ces émissions de poussières durent une vingtaine de minutes avec des concentrations nettement plus élevées durant les dix premières minutes, figure 2 . Les émissions ont été estimées à $1,92 \mathrm{~g} \cdot \mathrm{kg}^{-1}$ de bois sec ; les facteurs d'émissions, en fonction de l'allure de combustion et la classe d'humidité étant regroupés dans le tableau 1 . En allure réduite, figure 2, dès la réduction de l'arrivée de l'air (vers $50 \mathrm{mn}$ ), on note une augmentation rapide de la concentration en poussière. Les émissions avoisinent très souvent $2 \mathrm{~g} \cdot \mathrm{m}^{-3}$ pendant plusieurs heures et ne redescendent à des valeurs de 30 à $40 \mathrm{mg} \cdot \mathrm{m}^{-3}$ lorsqu'il ne reste plus que des résidus charbonneux.

Les émissions de poussières sont sensibles à l'humidité du bois, tableau 1. Pour du bois très sec (classe $\mathrm{C}_{1}$ ) les émissions de poussières avoisinent $2 \mathrm{~g} \cdot \mathrm{kg}^{-1}$; tandis que pour du bois sec (classe $\mathrm{C}_{2}$ ), ces émissions se voient multipliées par un facteur de 2,8 puisqu'elles ont été estimées à $5,75 \mathrm{~g} \cdot \mathrm{kg}^{-1}$. On notera en outre que le passage de l'allure nominale à l'allure réduite engendre lui aussi 3 fois plus de poussières. Enfin, du bois très humide (classe $\mathrm{C}_{3}$ ) engendre des concentrations de poussières nettement plus élevées, elles atteignent dans ce cas plus de $21 \mathrm{~g} \cdot \mathrm{kg}^{-1}$.

Quelques auteurs [1-4] ayant réalisé des études sur les $\mathrm{PM}_{10}$ (particules ayant un diamètre inférieur à $10 \mu \mathrm{m}$ ) ont abouti à des facteurs similaires qui s'étendent de 1 à $24 \mathrm{~g} \cdot \mathrm{kg}^{-1}$ de bois brûlé. Cependant, leurs études s'étendent à diverses technologies : foyers ouverts, poêles, inserts, poêles à granulés, diverses conditions opératoires et divers moyens de mesures. Une étude plus récente [5] portant sur les $\mathrm{PM}_{2,5}$ et $\mathrm{PM}_{0,9}$, révèle que les concentrations en $\mathrm{PM}_{2,5}$ varient entre 0,1 et 2,6 $\mathrm{g} \cdot \mathrm{kg}^{-1}$ de bois avec une moyenne de 1,3 et que les $\mathrm{PM}_{0,9}$ varient entre 0,03 et $6 \mathrm{~g} \cdot \mathrm{kg}^{-1}$ de bois avec une même moyenne de 1,3 .

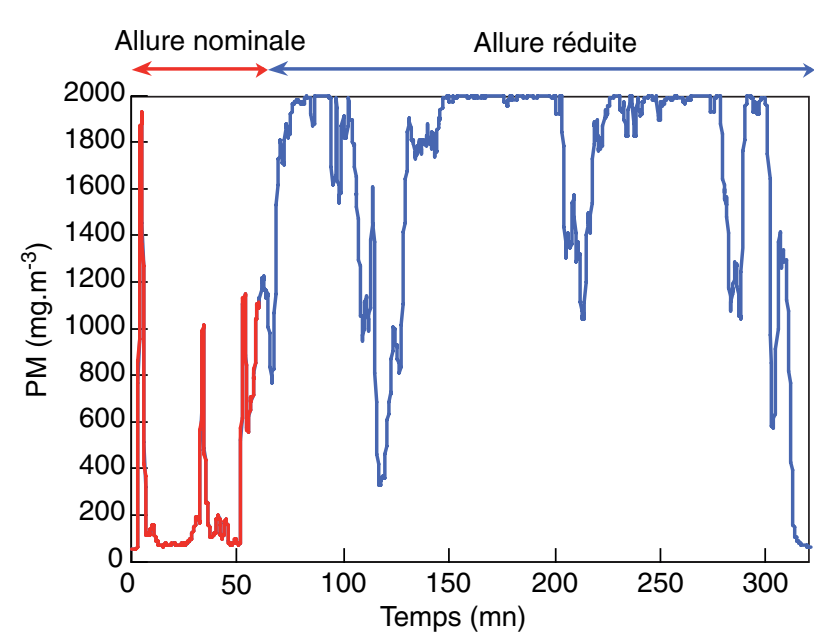

Figure 2

Émissions de poussière au cours de la combustion du bois différences entre allures nominale et réduite.

Example of dust emissions: comparison between a nominal and a reduced operation. 
TABLEAU I

Facteurs d'émissions des poussières en $\mathrm{g} \cdot \mathrm{kg}^{-1}$ de bois sec brûlé Dust expressed in $\mathrm{g} \mathrm{kg}^{-1}$ of seasoned wood

\begin{tabular}{c|c|c|c}
\hline Humidité & $\mathrm{C}_{1}$ & $\mathrm{C}_{2}$ & $\mathrm{C}_{3}$ \\
\hline \multicolumn{4}{c|}{ Allure nominale } \\
\hline Moyenne & - & 1,92 & - \\
\hline \multicolumn{4}{c}{ Allure réduite } \\
\hline Moyenne & 2,06 & 5,75 & 21,40 \\
\hline
\end{tabular}

En ce qui concerne la composition de ces poussières, elle dépend de beaucoup de facteurs. Une caractérisation physico chimique a été réalisée. Les poussières recueillies, voir figure 3 , ont une densité de 0,317 et un diamètre moyen de $13 \mu \mathrm{m}$ (écart type : 2,8 $\mu \mathrm{m}$ ) sur une étendue granulométrique de $0,2 \mu \mathrm{m}$ à $60 \mu \mathrm{m}$, comme montré à la figure 4 . Leur répartition en classe $\mathrm{PM}_{10}, \mathrm{PM}_{2,5}, \mathrm{PM}_{1}$ est respectivement de 30 , 10 , et $4 \%$. Ces résultats concernant la taille des poussières rendent capital, d'un point de vue environnemental et de santé public, l'approfondissement des connaissances de ce polluant solide susceptible de pénétrer si profondément les voies respiratoires de l'homme. En fait, ces poussières se composent de $36,5 \%$ de matières minérales et de $63,5 \%$ de matières volatiles. Une étude [6] sur la valorisation de cendres et poussières laisse entendre que les matières minérales se constituent généralement, selon les essences, de beaucoup de calcium et de silice, puis en plus petite quantité de fer, d'aluminium, de potassium, de phosphore, de manganèse, de magnésium, etc. Nos analyses révèlent qu'en terme de composition élémentaire les poussières se composent principalement de 40,9\% de carbone, de 2,7 \% d'hydrogène, de $3 \%$ d'azote et de $0,1 \%$ de soufre. Le fort niveau de carbone présent dans les poussières est le reflet d'une combustion non complète. En outre, les présences de HAP et de composés toxiques tels que le benzène dans les poussières ont été mises en évidence, tableau 2.

\section{TABLEAU 2}

Principaux hydrocarbures aromatiques contenus dans les poussières en $\mu \mathrm{g} \cdot \mathrm{kg}^{-1}$ de poussières

Aromatic hydrocarbons contained in dust expressed in $\mu \mathrm{g} . \mathrm{kg}^{-1}$ of dust

\begin{tabular}{l|c}
\multicolumn{1}{c|}{ Composés } & Concentration \\
\hline Phénanthrène & 8660 \\
Pyrène & 7660 \\
Acénaphtylène & 1300 \\
Naphtalène & 1200 \\
Biphényl & 900 \\
Benzène & 840 \\
Fluorène & 820 \\
Toluène & $<100$
\end{tabular}

Certains composés comme le benzène et certains HAP sont extrêmement toxiques (cancérigènes reconnus) même à faible concentration. Ces résultats démontrent que les poussières sont doublées d'un autre type de polluants à ne pas négliger : les hydrocarbures aromatiques. Plusieurs études $[7,8]$ confirment la présence notamment de benzène dans les fumées et les poussières à raison de $0,7 \mathrm{à} 0,95 \mathrm{~g}$ de benzène émis par kilogramme de bois brûlé ainsi qu'une forte concentration en divers HAP.

Le caractère extrêmement poreux des poussières [9] favorise l'adsorption de ces hydrocarbures ; ensuite ces poussières les véhiculent au plus profond du système respiratoire via l'atmosphère. Les problèmes de santé n'en seront que plus aigus pour l'homme et, au niveau environnemental,

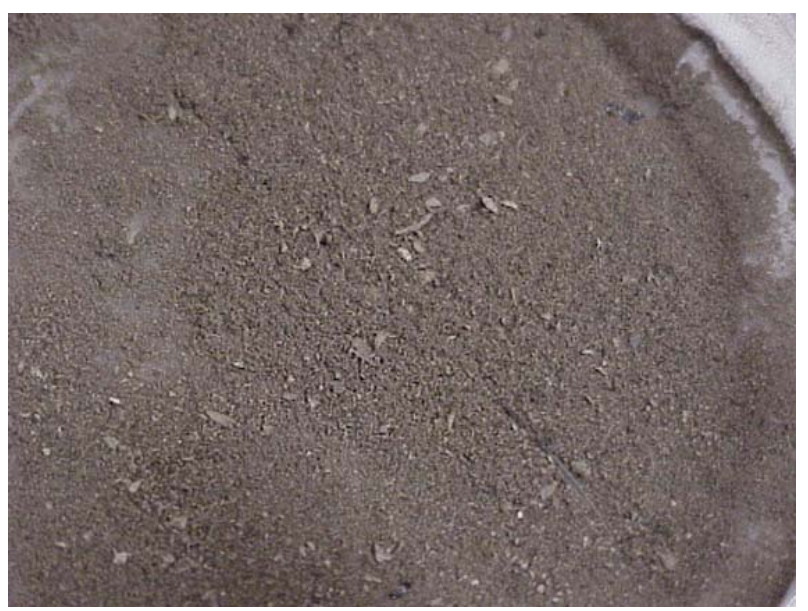

Figure 3

Poussières recueillies lors de la combustion de bois

Dusts emitted during firewood combustion

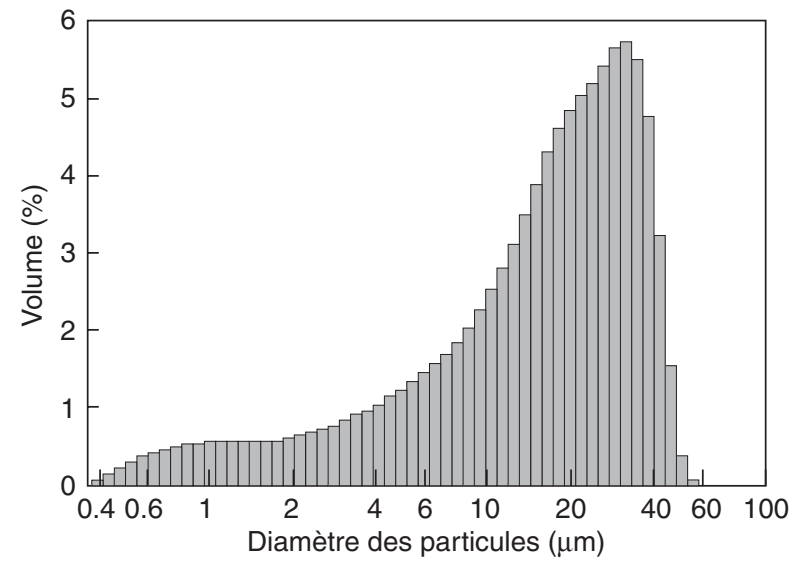

Figure 4

Répartition granulométrique des poussières. Granulometric distribution of dust. 
la présence de ces composés favorise les réactions photochimiques aboutissant à des composés polluants complexes donc difficiles à appréhender et à traiter.

Outre ces composés, les matières volatiles renferment des acides organiques, tel que l'acide acétique qui reflètent le côté corrosif des poussières. Les principaux acides sont présentés dans le tableau 3.

\section{TABLEAU 3}

Principaux acides organiques contenus dans les poussières en $\mathrm{mg} \cdot \mathrm{kg}^{-1}$ de poussières

Organic acids contained in dust expressed in $\mathrm{mg} \cdot \mathrm{kg}^{-1}$ of dust

\begin{tabular}{l|c}
\hline Principaux acides gras & Concentration \\
\hline Acide acétique & 3570 \\
Acide lactique & 2830 \\
Acide formique & 1290 \\
Acide propionique & 205 \\
Acide butyrique & 7,5 \\
Acide valérique & $<5$ \\
\hline
\end{tabular}

\section{2 Émissions de polluants gazeux}

\subsubsection{Composés imbrûlés}

Issus d'une mauvaise combustion ou d'une combustion incomplète les polluants carbonés tels que le monoxyde de carbone ou certains hydrocarbures, représentent les principaux polluants gazeux générés par la combustion du bois.

La formation de $\mathrm{CO}$ au cours de la combustion du bois intervient comme élément perturbateur des réactions chimiques de combustion, tant sur l'aspect libération d'énergie que sur l'utilisation de l'oxygène. De plus, ce polluant est extrêmement toxique. Très rapidement absorbé par les poumons, le CO se fixe sur l'hémoglobine dont l'affinité pour le monoxyde de carbone est 240 fois supérieure à celle pour l'oxygène. L'intoxication au monoxyde de carbone peut entraîner une mort rapide. Les émissions de CO, au cours de la combustion du bois, sont très importantes lors de la phase de démarrage, alors que la combustion est très hétérogène, figure 5. Elles atteignent près de 10000 ppmv (1\%). Mais dès que la combustion s'homogénéise (visible par la présence de flammes conséquentes) une baisse sensible des émissions de $\mathrm{CO}$ apparaît de façon nette. A ce stade les émissions de $\mathrm{CO}$ ne dépassent guère 0,3 à $0,5 \%$. Par contre, dés que la température chute et de ce fait qu'apparaît la phase de combustion des résidus charbonneux, les émissions de $\mathrm{CO}$ ré-augmentent et ce jusqu'à atteindre des valeurs de 0,6 à $0,9 \%$ lors de fonctionnements en allure nominale et des valeurs de 1 à $1,5 \%$ pour des fonctionnements en allure réduite.

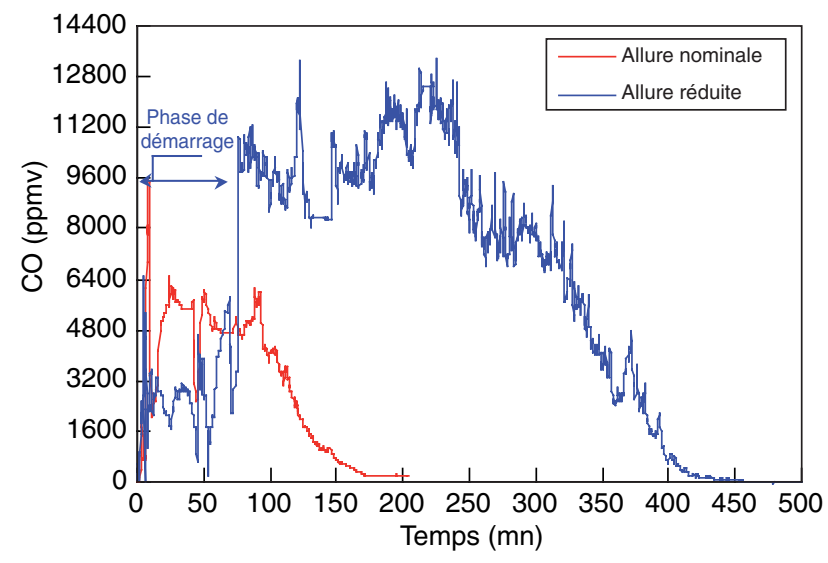

Figure 5

Émissions de $\mathrm{CO}$ au cours de la combustion du bois différences entre allures nominale et réduite.

Example of $\mathrm{CO}$ emissions: comparison between a nominal and a reduced operation.

\section{TABLEAU 4}

Facteurs d'émissions du monoxyde de carbone en $\mathrm{g} \cdot \mathrm{kg}^{-1}$ de bois sec brûlé

Emission of carbon monoxide expressed in $\mathrm{g}^{\mathrm{kg}} \mathrm{kg}^{-1}$ of seasoned wood

\begin{tabular}{c|c|c|c}
\hline Humidité & $\mathrm{C}_{1}$ & $\mathrm{C}_{2}$ & $\mathrm{C}_{3}$ \\
\hline \multicolumn{4}{c}{ Allure nominale } \\
\hline Moyenne & - & 79 & - \\
\hline \multicolumn{4}{c}{ Allure réduite } \\
\hline Moyenne & 79 & 168 & 274 \\
\hline
\end{tabular}

Les émissions de $\mathrm{CO}$ sont sensibles à tout paramètre influençant la combustion. Il ressort des résultats présentés, tableau 4, que le passage de l'allure nominale à l'allure réduite, pour une même classe d'humidité, en l'occurrence $\mathrm{C}_{2}$, double les émissions de CO. La réduction de l'arrivée d'air favorise une combustion incomplète avec production très importante de $\mathrm{CO}$.

L'augmentation de l'humidité du combustible est également préjudiciable à la quantité de $\mathrm{CO}$ produite. En effet, on aboutit à deux fois plus de $\mathrm{CO}$ produit lorsque l'on brûle du bois sec de classe $\mathrm{C}_{2}, 168 \mathrm{~g} \cdot \mathrm{kg}^{-1}$, par rapport à du bois très sec de classe $C_{1}, 79 \mathrm{~g} \cdot \mathrm{kg}^{-1}$; et un peu moins de quatre fois plus lorsqu'on utilise du bois humide : $274 \mathrm{~g} \cdot \mathrm{kg}^{-1}$.

Ces conclusions, quant à l'influence de l'allure de combustion et à l'humidité du combustible, restent valables pour les émissions de $\mathrm{HC}$, tableau 5. En effet, tout comme le CO, on obtient près de deux fois plus de $\mathrm{HC}$ produit lorsque l'on brûle du bois sec de classe $\mathrm{C}_{2}, 3,8 \mathrm{~g} \cdot \mathrm{kg}^{-1}$, par rapport à du bois très sec de classe $\mathrm{C}_{1}, 2,1 \mathrm{~g} \cdot \mathrm{kg}^{-1}$; et plus de quatre fois plus lorsqu'on utilise du bois humide : $9,1 \mathrm{~g} \cdot \mathrm{kg}^{-1}$. Il a d'ailleurs pu être mis en évidence une certaine corrélation entre émissions de $\mathrm{CO}$ et $\mathrm{HC}$. 
TABLEAU 5

Facteurs d'émissions des hydrocarbures totaux en $\mathrm{g} \cdot \mathrm{kg}^{-1}$ de bois sec brûlé

Emission of hydrocarbon expressed in $\mathrm{g} \cdot \mathrm{kg}^{-1}$ of seasoned wood

\begin{tabular}{c|c|c|c}
\hline Humidité & $\mathrm{C}_{1}$ & $\mathrm{C}_{2}$ & $\mathrm{C}_{3}$ \\
\hline \multicolumn{4}{c}{ Allure nominale } \\
\hline Moyenne & - & 2,4 & - \\
\hline \multicolumn{4}{c}{ Allure réduite } \\
\hline Moyenne & 2,1 & 3,8 & 9,10 \\
\hline
\end{tabular}

Un prélèvement ponctuel des fumées a permis de réaliser une recherche de quelques HAP dans les fumées. La présence de 15 HAP a été mis en évidence dans les fumées, tableau 6 ; les principaux étant le pyrène, la naphtalène, le phénanthrène et le benzo(a)anthracène.

\section{TABLEAU 6}

Principaux hydrocarbures aromatiques polycycliques issus de la combustion du bois en $\mathrm{ng} \cdot \mathrm{Nm}^{-3}$

Polycyclic aromatic hydrocarbon expressed in $\mathrm{ng} \cdot \mathrm{Nm}^{-3}$

\begin{tabular}{l|c}
\hline Acénaphtylène & 175 \\
Anthracène & 168 \\
Benzo (a) anthracène & 3570 \\
Benzo (b) fluoranthène & 2160 \\
Benzo (k) fluoranthène & 2976 \\
Benzo (ghi) pérylène & 3038 \\
Benzo (a) pyrène & 2909 \\
Chrysène & 2991 \\
Dibenzo-ah-anthracène & 381 \\
Fluoranthène & 635 \\
Fluorène & 117 \\
Indéno-Pyrène & 1183 \\
Naphtalène & 4629 \\
Phénanthrène & 3358 \\
Pyrène & 5559 \\
\hline
\end{tabular}

\subsubsection{Autres polluants}

Afin de compléter cette caractérisation des polluants émis lors de la combustion du bois, les oxydes d'azote et de soufre ont été recherchés.

Les oxydes d'azote peuvent provenir de l'azote contenu dans l'air comburant ou de l'azote contenu dans le combustible. Les oxydes d'azote provenant de l'air se forment à très haute température en tant qu'oxydes d'azote dits thermiques. Dans les chauffages au bois habituels, les températures de combustion étant inférieures à $1300{ }^{\circ} \mathrm{C}$, les NOx thermiques revêtent une importance minime. Par contre, le bois contient de l'azote qui dans l'arbre se présente sous la forme d'amines et de protéines nécessaires à sa croissance ; c'est l'oxydation de cet azote qui produira, en fonction de l'essence, la majeure partie de NOx émis. Ce sont les NOx « combustibles » [10]. Lors de la combustion, l'azote commence par se dégager sous forme de molécules azotées de faible poids moléculaire $\left(\mathrm{NH}_{3}\right)$ ou de radicaux libres $\left(\mathrm{NH}_{2}, \mathrm{NH}, \mathrm{HCN}, \mathrm{CN}\right)$, qui jouent un rôle de précurseur dans la formation des NOx. Les liaisons de l'azote avec ces autres éléments, carbone, hydrogène, sont des liaisons relativement faibles. En conséquence, la formation des $\mathrm{NO}$ « combustible » se fait même à des températures limitées, par contre, elle nécessite la présence d'oxygène en excès dans la zone de combustion. Il est très difficile d'estimer la proportion d'azote qui se voit transformer au cours de la combustion, en NOx. Le « taux de conversion », c'est-à-dire la masse d'azote contenue initialement dans le combustible rapportée à celle que l'on trouve sous forme de NOx varie entre 25 et $75 \%$. Par contre, dans un foyer donné, la modification, en plus ou en moins, de la teneur en azote du combustible utilisé se traduit immanquablement par une variation de la teneur en $\mathrm{NO}_{x}$ dans les fumées.

Les facteurs d'émissions des NOx obtenus suite aux mesures effectuées, sont de l'ordre de $0,35 \mathrm{~g} \cdot \mathrm{kg}^{-1}$ pour du bois de classe $\mathrm{C}_{2}$ en allure nominale. Cette valeur relativement faible n'a pas conduit à un approfondissement des mesures.

Quant aux oxydes de soufre, il n'est apparu que des traces, jugées insignifiantes.

\section{CONCEPTS ÉPURATEURS}

L'objectif de cette recherche est de pouvoir proposer un nouveau concept de conduit de fumée « épurateur » qui par l'action combinée de plusieurs procédés serait susceptible de réduire, d'éliminer, ou de traiter les polluants visés. Au vu des résultats précédents, on va s'attacher à proposer un système visant à abattre les poussières, le monoxyde de carbone et les hydrocarbures imbrûlés. Même si l'objectif est d'aboutir à un seul prototype, à ce jour, ont été étudiés séparément, l'épuration des poussières et celle conjointement, du monoxyde de carbone et des hydrocarbures imbrûlés.

\section{1 Élimination des poussières}

Compte tenu des contraintes liées aux particules (taille, densité, concentration), des contraintes liées au vecteur fumées (débit, température, pression, point de rosée) et celles liées au procédé lui-même (rendement, encombrement, entretien, coûts) et après l'étude des différents phénomènes physiques susceptibles de participer à la séparation de particules solides (filtration, impaction, gravité, sédimentation), il ressort que l'effet centrifuge semble le procédé le plus judicieux à mettre en œuvre. 


\subsubsection{Banc de simulation}

Un banc de test pour les séparateurs de poussières a été conçu dans le but d'optimiser l'étude de différents concepts épurateurs. Il s'agit de mettre au point un outil générant de la poussière de façon homogène tout en reproduisant les conditions aérauliques des conduits de fumées. Ce banc, figure 6, permet d'estimer l'efficacité de prototypes de séparation et de pouvoir étudier l'influence de divers paramètres tels que la concentration en poussières ou la vitesse en entrée ainsi que différents paramètres géométriques, propres au séparateur. Un système de tamis chargé d'une masse de poudre fixée, actionné par un moteur, injecte de façon homogène cette poudre dans une canalisation. La poudre, du polymère $64 \mathrm{~B}$ 87 a été choisie pour ces caractéristiques proches de celles des poussières (masse volumique : $0,52 \mathrm{~g} \cdot \mathrm{cm}^{-3}$ et granulométrie de 10 à $80 \mu \mathrm{m}$ avec un diamètre moyen de 26,5 $\mu \mathrm{m}$ ). Par le biais d'un ventilateur à régime variable placé en fin de ligne, cette poudre est aspirée à travers des éléments de conduits et le prototype de séparation à tester. La vitesse en entrée peut varier de 0 à $4 \mathrm{~m} \cdot \mathrm{s}^{-1}$. Dans un premier temps il a été choisi de travailler entre 0,5 et $1,4 \mathrm{~m} \cdot \mathrm{s}^{-1}$ afin de se rapprocher de la vitesse usuellement rencontrée dans les conduits de fumées. Un filtre HEPA en fin de banc permet de récupérer l'intégralité des poussières véhiculées dans le montage ; son

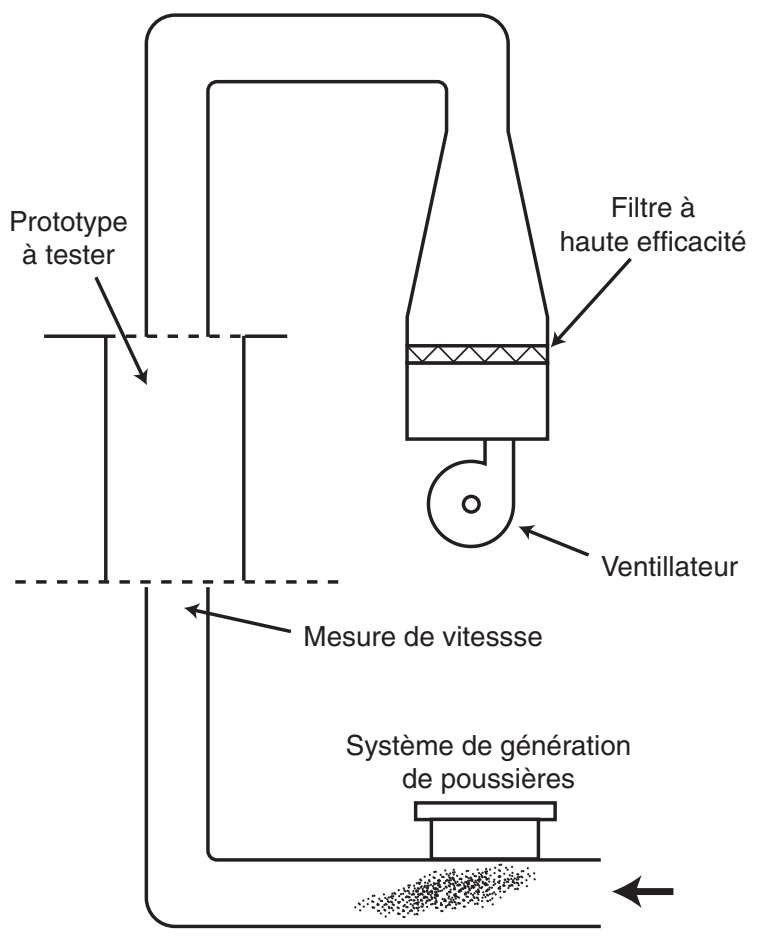

Figure 6

Principe du banc de test des séparateurs de poussières. Experimental bench to simulate dust generation.

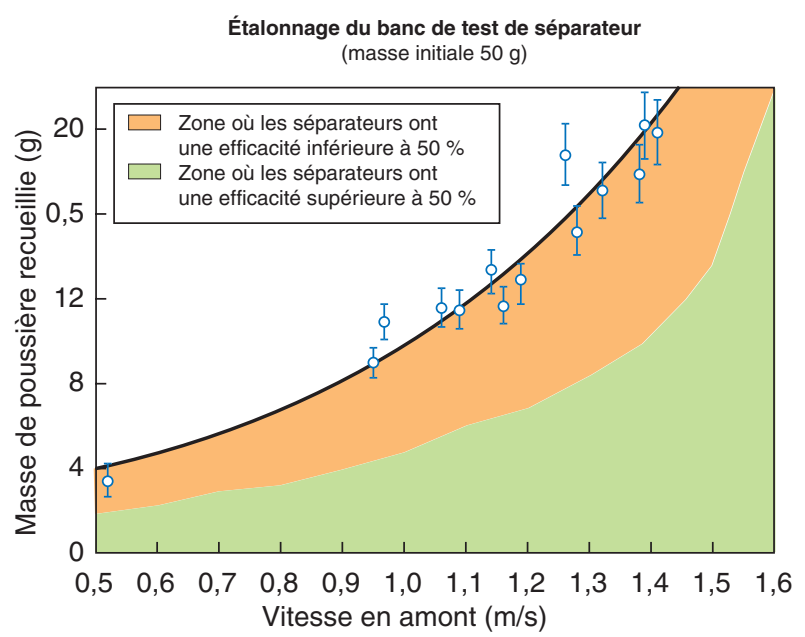

Figure 7

Courbe d'étalonnage du banc de test.

Calibration curve for the dust bench.

seuil de coupure étant de $0,3 \mu \mathrm{m}$. Le principe de mesure actuel repose sur une pesée de la masse introduite avant le prototype et une pesée de la masse recueillie sur le filtre après le prototype de séparation. Il a donc été nécessaire de connaître la réponse du banc, sans aucun prototype afin de pouvoir estimer les pertes de poussières dues au montage (fuites, singularités, zones d'accumulation) et d'obtenir en fonction de la vitesse amont, une courbe de référence. Cette courbe d'étalonnage est présentée figure 7. L'évolution de la masse de poussière de référence $\left(M_{r e f}\right)$ recueillie sur filtre évolue de façon exponentielle avec la vitesse (v). Une loi de régression obtenue par la méthode des moindres carrés permet d'obtenir la relation suivante (1) :

$$
M_{r e f}=1,8753 \cdot \mathrm{e}^{1,6884 . \mathrm{v}}
$$

Cette courbe va permettre d'estimer l'efficacité de séparation d'un prototype testé. En effet, pour une vitesse donnée, on compare la masse de poussières de référence $\left(M_{r e f}\right)$ recueillie sur le filtre à celle recueillie lorsque le séparateur est en place $(M)$.

$$
E=\frac{M_{r e f}-M}{M_{r e f}} \times 100
$$

\subsubsection{Séparateur de poussière}

Après diverses mises en œuvre de l'effet centrifuge pour séparer les poussières dans les conduits de fumées : cyclone, vis fixe [11,12], notre développement s'est orienté vers un centrifugeur mécanique, figure 8 . Une pâle de $500 \mathrm{~mm}$ de longueur et de $156 \mathrm{~mm}$ de largeur reliée à un axe de rotation 


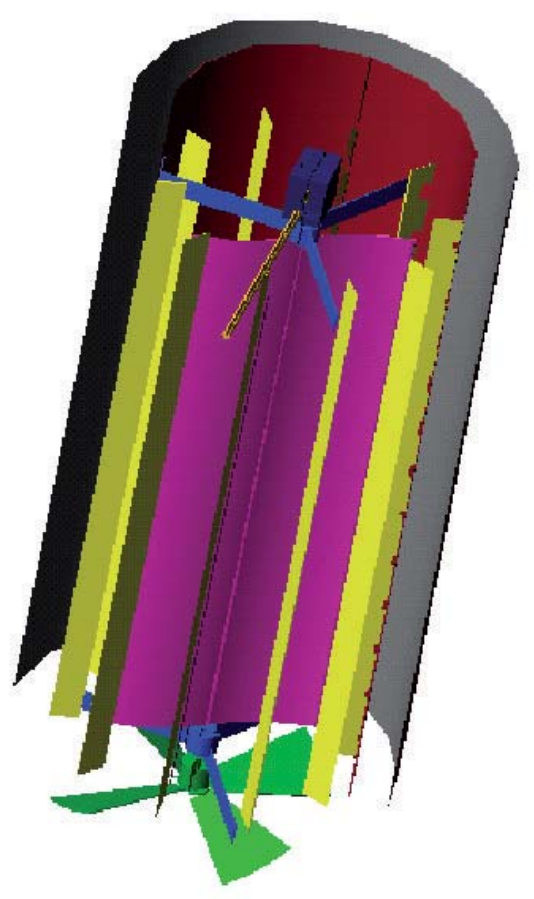

Figure 8

Schéma de principe du prototype de séparation de poussières. Plan of the prototype of dusts separation.

est installée dans un élément de conduit double peau de $200 \mathrm{~mm}$ de diamètre intérieur. Cette pâle est entrânée par un moteur électrique de $12 \mathrm{~V}$ dont la vitesse de rotation avoisine $1400 \mathrm{tr} \cdot \mathrm{mn}^{-1}$. Ce moteur est à l'extérieur du conduit. Une étude de sensibilité concernant l'influence de la vitesse de rotation de la pâle sur l'efficacité du procédé a été réalisée. Il s'avère que plus la vitesse de rotation est importante meilleure est l'efficacité du système. Cependant, compte tenu de la stabilité des pièces d'entrainement (arbre, engrenage) et du bruit occasionné, la vitesse de $1400 \mathrm{tr} \cdot \mathrm{mn}^{-1}$ semble être un bon compromis. Huit ailettes verticales de $500 \mathrm{~mm}$ de longueur et de $20 \mathrm{~mm}$ de largeur équipent l'intérieur du conduit de façon à favoriser une zone d'accumulation des poussières projetées par la pâle sur la paroi. Les poussières retombent par gravité dans une gouttière en amont de la pâle. L'élément de conduit suivant ce prototype a été également équipé d'ailettes de mêmes dimensions que les premières, dans le but d'optimiser la rétention de poussières. Des photos réalisées lors des essais sur banc de simulation sont disponibles figure 9

L'efficacité du prototype testé sur banc de simulation, avoisine un captage de particules de $56 \%$ sur la gamme de vitesse testée. Les résultats expérimentaux sont présentés figure 10. Il ressort que l'efficacité est relativement constante sur la gamme de vitesse testée représentant celle des fumées; elle évolue entre 40 et $74,5 \%$. Encouragés par ces résultats, le prototype a été usiné de façon à être installé dans un conduit de fumée réel. Afin d'être le moins possible soumis aux contraintes thermiques, le moteur a été placé à l'extérieur du conduit. Si les résultats de ce prototype de séparation en situation réelle étaient probants, des solutions comme le développement d'un moteur Stirling serait par la suite envisageable. Ne perdant pas de vue le fait que ce séparateur de poussières sera couplé à un autre procédé d'épuration, lors de sa conception, une hélice a été rajoutée. Elle a pour but de vaincre les pertes de charge occasionnée par le système prévu pour la réduction du $\mathrm{CO}$ et des $\mathrm{HC}$. Il s'agit d'une hélice à quatre aubes ayant une inclinaison de $25^{\circ}$.

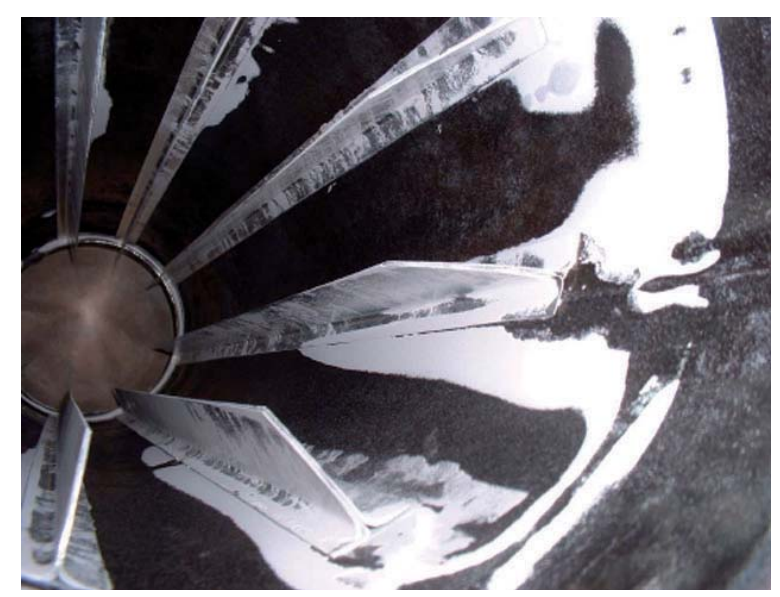

Figure 9

Rétention des poussières par les ailettes verticales. Dusts retained by the vertical fins.

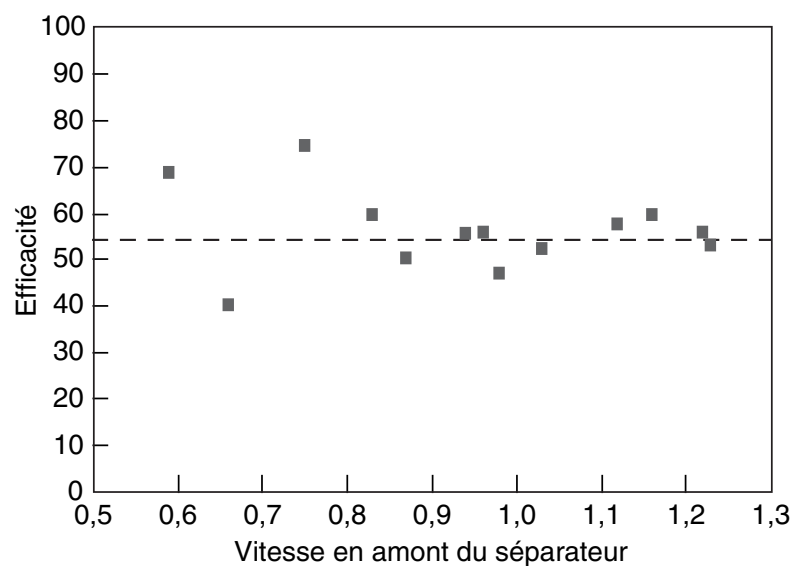

Figure 10

Efficacité du prototype de séparation de poussières - résultats obtenus sur banc de simulation.

Efficiency of dust separation prototype - results obtained on dust bench. 


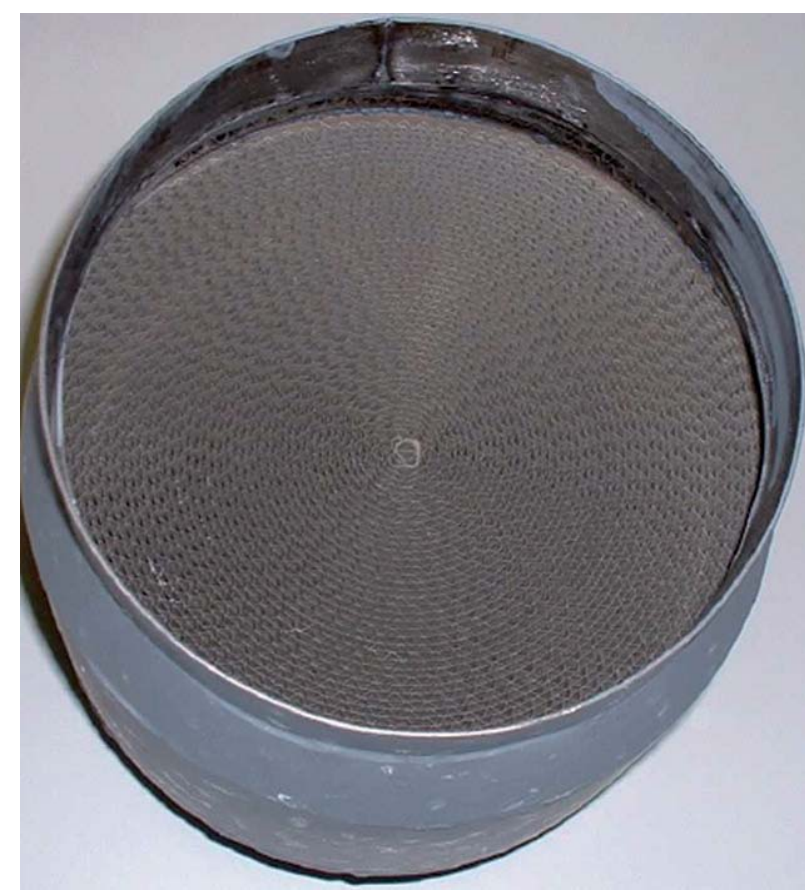

Figure 11

Catalyseur mis en place pour les essais d'oxydation des $\mathrm{CO}$ et $\mathrm{HC}$.

Catalyst for the oxydation of $\mathrm{CO}$ et $\mathrm{HC}$.

\subsection{Oxydation du $\mathrm{CO}$ et des $\mathrm{HC}$}

\subsubsection{Le catalyseur}

Pour ce qui est de l'épuration des $\mathrm{CO}$ et $\mathrm{HC}$, l'oxydation catalytique se révèle être l'un des moyens les plus efficaces [13-16]. C'est pourquoi l'emploi d'un catalyseur comme épurateur des $\mathrm{CO}$ et $\mathrm{HC}$ dans un conduit de fumées a été étudié. Il s'agit d'un catalyseur à base de platine inséré dans un conduit de fumées en sortie d'un insert à bois . Le catalyseur testé a été conçu par les procédés Percevaut, figure 11. La partie active de ce catalyseur est un élément cylindrique de diamètre $250 \mathrm{~mm}$ et de hauteur $200 \mathrm{~mm}$. Il comporte un enroulement des plaques ondulées en acier inox réfractaire revêtues d'un support d'alumine sur lequel est déposé du platine ; il et est destiné à traiter des fumées contenant des teneurs moyennes de l'ordre de 4000 ppmv en $\mathrm{CO}$ et 1000 ppmv en HC. Les essais ont été réalisés avec un insert à bois, utilisé en allure nominale avec des bûches de chêne de classe $\mathrm{C}_{2}$. Le conduit de fumée sur lequel a été installé le catalyseur a été instrumenté de façon à pouvoir mesurer en continu, avant et après le catalyseur, les concentrations en $\mathrm{CO}, \mathrm{HC}$ et $\mathrm{CO}_{2}$. La période de basculement des électrovannes assurant les mesures amont/aval des polluants a été fixée à 1 minute. Pour des raisons de sécurité et afin de ne pas endommager le catalyseur au cours des phases de démarrage, le conduit de fumées a été muni d'un by-pass permettant d'orienter ou non les fumées dans le catalyseur. Généralement pendant les 10 premières minutes d'essai, les fumées sont déviées avant de passer par le catalyseur. À noter que le by-pass reste continuellement ouvert de $10 \%$ et ce pour des raisons de sécurité. Avant son insertion dans le conduit, une étude en soufflerie a permis d'estimer la perte de charge du catalyseur. Pour une vitesse de $1 \mathrm{~m} \cdot \mathrm{s}^{-1}$, la perte de charge occasionnée par le catalyseur est de 3 mbar.

\subsubsection{Résultats expérimentaux}

Les tableaux 7 et 8 reprennent l'ensemble des résultats relatifs à l'abattement des $\mathrm{CO}$ et $\mathrm{HC}$ résultant de l'emploi d'un catalyseur au platine dans un conduit de fumées relié à un appareil au bois. Tant que les températures minimales de fonctionnement du catalyseur ne sont pas atteintes, soit environ $200^{\circ} \mathrm{C}$ pour oxyder le $\mathrm{CO}$, le flux de fumées est évacué par un conduit parallèle. Il a donc été nécessaire de définir plusieurs rendements d'abattement. Un premier rendement d'abattement concernant la durée totale de la combustion est appelé rendement global, $\eta_{\mathrm{g}}$; un second rendement relatif à la période d'efficacité du catalyseur pour le composé visé est appelé rendement catalyseur, $\eta_{\text {cat }}$. 12 essais ont été réalisés, pour chacun les charges de bois variaient entre 6 et $10 \mathrm{~kg}$.

TABLEAU 7

Rendement d'abattement de CO avec l'emploi d'un catalyseur Efficiency of $\mathrm{CO}$ decrease by using catalyst

\begin{tabular}{c|c|c|c|c}
\cline { 2 - 5 } & \multicolumn{2}{c|}{ Durée } & \multicolumn{2}{c}{ Rendement } \\
\cline { 2 - 5 } & Combustion & $\begin{array}{c}\text { Fonctionnement } \\
\text { catalyseur }\end{array}$ & Global & Catalyseur \\
\cline { 2 - 5 } & $\mathrm{mn}$ & $\mathrm{mn}$ & $\%$ & $\%$ \\
\hline Moyenne & 325 & 178 & 56 & 73 \\
Maximum & 410 & 300 & 85 & 90 \\
Minimum & 270 & 55 & 20 & 54 \\
Écart type & 44 & 99 & 27 & 15 \\
\hline
\end{tabular}

Une moyenne de $73 \%$ du CO est oxydée lors de son passage par le catalyseur ; il s'agit du rendement « catalyseur ». $\mathrm{Si}$ au contraire on considère la durée totale de la combustion comme référence, le rendement global moyen est abaissé à $56 \%$. Il peut varier entre 20 et $85 \%$. Les expérimentations nous ont permis de constater que lorsque la durée de fonctionnement du catalyseur est inférieure à $100 \mathrm{mn}$, les rendements sont plus faibles : $\eta_{\mathrm{g}}$ varie entre 20 et $39 \%$ et $\eta_{\text {cat }}$ entre 54 et $64 \%$. Tandis que pour des fonctionnements plus longs, supérieurs à $150 \mathrm{mn}, \eta_{\mathrm{g}}$ varie entre 47 et $85 \%$ et $\eta_{\text {cat }}$ entre 68 et $90 \%$. La température nécessaire à la conversion de prés de $90 \%$ du $\mathrm{CO}$ est de l'ordre de $200{ }^{\circ} \mathrm{C}$, figure 12 . Les 
Combustion d'une charge de bois dans un insert classique

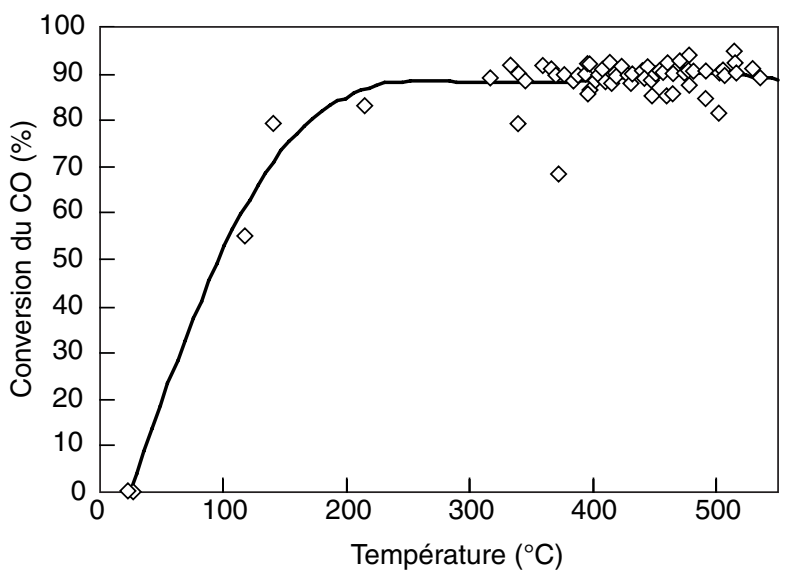

Figure 12

Conversion du $\mathrm{CO}$ en fonction de la température.

CO conversion with temperature.

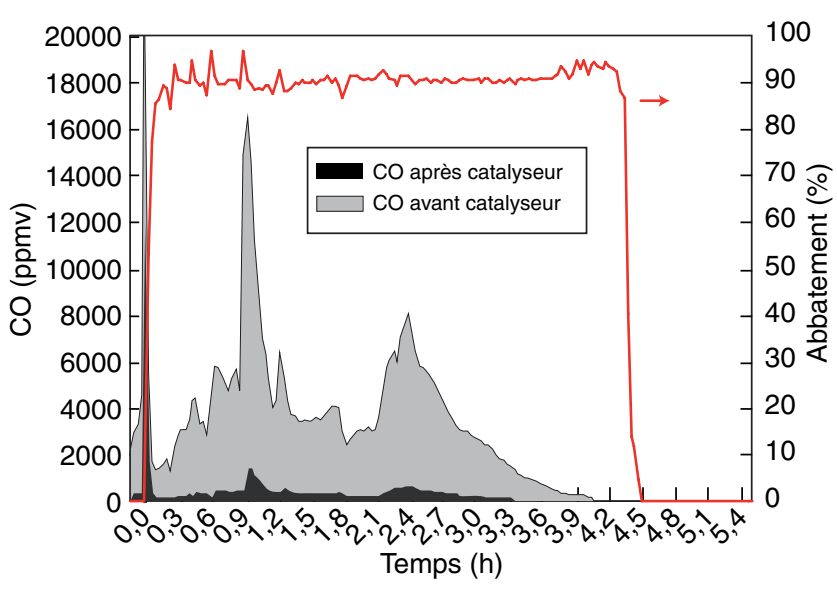

Figure 13

Évolution du $\mathrm{CO}$ avant et après catalyseur - courbe de rendement. CO evolution before and after the catalyst - efficiency curves. réactions catalytiques étant exothermiques, le catalyseur s'échauffe et son inertie thermique permet une conversion du $\mathrm{CO}$ même lorsque les températures de fumées sont redescendues aux environs de 80 à $70{ }^{\circ} \mathrm{C}$. C'est pourquoi, plus la durée de fonctionnement du catalyseur est importante, meilleur est son rendement d'élimination. Il a été possible d'atteindre jusqu'à $90 \%$ d'élimination de $\mathrm{CO}$ et ce sur plus de la moitié de la durée totale de la combustion, figure 13. Des travaux visant à améliorer le fonctionnement du catalyseur lors des phases de démarrage restent à faire ; ils permettraient d'augmenter le rendement global ainsi que la durée de fonctionnement du catalyseur. Peu d'études peuvent servir de référence sur ce point précis [17].

\section{TABLEAU 8}

Rendement d'abattement des $\mathrm{HC}$ avec l'emploi d'un catalyseur Efficiency of HC decrease by using catalyst

\begin{tabular}{c|c|c|c|c}
\cline { 2 - 5 } & \multicolumn{2}{|c|}{ Durée } & \multicolumn{2}{c}{ Rendement } \\
\cline { 2 - 5 } & Combustion & $\begin{array}{c}\text { Fonctionnement } \\
\text { catalyseur }\end{array}$ & Global & Catalyseur \\
\cline { 2 - 5 } & $\mathrm{mn}$ & $\mathrm{mn}$ & $\%$ & $\%$ \\
\hline Moyenne & 325 & 178 & 27 & 37 \\
Maximum & 410 & 300 & 44 & 46 \\
Minimum & 270 & 55 & 15 & 29 \\
Écart type & 44 & 99 & 9 & 7 \\
\hline
\end{tabular}

En ce qui concerne les $\mathrm{HC}, \eta_{\mathrm{g}}$ atteint $44 \%$ pour une moyenne de $27 \%$ et $\eta_{\text {cat }}$ ne dépasse pas $46 \%$ pour une moyenne de $37 \%$. On constate que l'oxydation des $\mathrm{HC}$ reste plus compliquée à obtenir. En effet, la température nécessaire à cette oxydation, 450 à $600{ }^{\circ} \mathrm{C}$, étant supérieure aux températures des fumées, initialement seuls quelques hydrocarbures facilement oxydables réagissent au passage du catalyseur. Le dégagement de chaleur interne du catalyseur va permettre d'augmenter cette transformation sans pour autant obtenir les mêmes taux de conversion que pour le CO.

L'augmentation du dégagement de $\mathrm{CO}_{2}$ a été mesurée. $\mathrm{La}$ moyenne d'augmentation de $\mathrm{CO}_{2}$ lors du fonctionnement du catalyseur est de $9 \%$; elle varie pour l'ensemble des 12 essais, entre 2 et $14 \%$.

\section{CONCLUSION}

La finalité des travaux présentés dans cet article est de développer un système d'épuration adapté aux conduits de fumées ; système qui, tout en permettant d'évacuer les produits de combustion en toute sécurité, permettrait de réduire la pollution engendrée par la combustion, en l'occurrence du bois.

La première partie de ces travaux concerne donc la caractérisation des fumées en terme de rejet de polluants et ce, à l'issue d'un insert à bois. Cette étude préliminaire revêt une importance primordiale dans le choix et les orientations à prendre, en terme d'épuration. Il a été mis en évidence l'émission significative de poussière, entre 1,9 à $21 \mathrm{~g} / \mathrm{kg}$ de bois sec brûlé. En plus de leur caractère pollueur propre, il a été mis en évidence que ces particules solides véhiculaient du benzène et différents HAP, substances répertoriées comme cancérigènes avérés ou probables. Les émissions de $\mathrm{CO}$ et $\mathrm{HC}$ sont parmi les plus significatives. En ce qui concerne le CO elles varient entre 79 et $274 \mathrm{~g} \cdot \mathrm{kg}^{-1}$ de bois sec brûlé, et pour les hydrocarbures totaux entre 2,1 et $9,1 \mathrm{~g} . \mathrm{kg}^{-1}$ de bois sec brûlé.

Il a donc été décidé de s'orienter dans deux voies d'épuration : la séparation de poussières et la réduction des polluants gazeux carbonés que sont le monoxyde de carbone 
et les hydrocarbures imbrûlés. Pour ce qui est des poussières, l'effet centrifuge a été retenu et, adapté aux contraintes rencontrées dans les conduits de fumées. Il offre des résultats encourageants sur banc d'essai entre 50 et $60 \%$. En ce qui concerne la réduction des polluants gazeux tels que le monoxyde de carbone et les hydrocarbures, le choix s'est porté sur l'oxydation catalytique. Cette voie d'épuration a démontré de réels résultats puisque les rendements moyens d'abattement de $\mathrm{CO}$ sont de l'ordre de 75 à $90 \%$ et de 29 à $46 \%$ pour les HC pour le seul fonctionnement du catalyseur. Les rendements globaux, basés sur la durée totale de la combustion sont légèrement inférieurs.

De nombreuses réflexions restent à mener pour optimiser le fonctionnement de ces deux systèmes d'épuration, notamment lors de leur futur fonctionnement conjoint. Le développement d'un tel concept épurateur ne doit en aucun cas perturber le bon fonctionnement du conduit de fumées. C'est pourquoi différents aspects tels que la mise en œuvre, la consommation électrique, la facilité d'entretien et ramonage, le risque d'intoxication au monoxyde de carbone entre autre, doivent être étudiés, ainsi que le vieillissement du catalyseur. C'est principalement dans ce sens que cette recherche doit se poursuive.

\section{RÉFÉRENCES}

1 de Angelis D. and Hall R. (1980) EPA's research program for controling residential wood combustion emissions, Journal of the air pollution control association, 30, 8, 862-867.

2 Butcher S. and Sorenson E. (1979) A study of wood stove particulate emissions, Journal of the air pollution control association, 29, 7, 724-728.

3 Dasch J.M. (1982) Particulate and gaseous emissions from wood burning fireplaces, Environmental science and technology, 16,10 .
4 EPA (1996) Residential wood stoves, Report on revision to 5 th edition AP-42, Section 1-10.

5 Hedberg E. (1994) Extension de la méthode des quadripôles thermiques à l'aide de transformations intégrales, Int. J. Heat Mass Trans. 1, 111-127.

6 ADEME-DVNAC (2001) Etude de la valorisation des cendres de chaufferies bois.

7 Butcher S. and Ellenbecker M. (1982) Particulate emission factors for small wood and coal stoves, Journal of the air pollution control association, 32, 4, 380-384.

8 Hueglin C.H. and Gaegauf C.H. (1997) Characterization of wood combustion particles: morphology, mobility and photoelectric activity, Environmental science and technology, 31, 12, 3439-3447.

9 Osan J. (2002) Characteriszation of wood combustion particles using electron probe microanalysis, atmospheric environment, 36, 2207-2214.

10 Jacubowiez I., Dénitrification des gaz de combustion, Techniques de l'ingénieur, J3922.

11 Robert L. (2003) L'épuration des poussières issues de la combustion domestique au bois, Cahiers du CSTB, 3475.

12 Robert L. (2004) Epuration des poussières issues de la combustion domestique au bois, COFRET'04, Nancy.

13 Wang H., Xiao T., Su J., Liu W. and Lu Y. (1999) Catalytic purification of flue gas from civil_used stove, Catalysis Today, 53, 661-667.

14 Carnö J. and Järäs S. Catalytic abatement of emissions from small-scale combustion of wood, a comparison of the catalytic effect in model and real flue gases, Fuel, 75, 8, 959-965.

15 Corning catalytic combustion, Design Handbook, Industrial Materials Department, Corning, New York 14831.

16 Saracco G. and Specchia V. (2000) Catalytic filters for the abatment of volatile organic compounds, Chemical Engineering Science, 55, 897-908.

17 Ferrandon M., Berg M. and Björnbom E. (1999) Thermal stability of metal-supported catalysts for reduction of cold-start emissions in a wood-fired domestic boiler, Catalysis Today, 53, 647-659.

Final manuscript received in January 2006

Copyright $(\mathbb{C} 2006$ Institut français du pétrole

Permission to make digital or hard copies of part or all of this work for personal or classroom use is granted without fee provided that copies are not made or distributed for profit or commercial advantage and that copies bear this notice and the full citation on the first page. Copyrights for components of this work owned by others than IFP must be honored. Abstracting with credit is permitted. To copy otherwise, to republish, to post on servers, or to redistribute to lists, requires prior specific permission and/or a fee: Request permission from Documentation, Institut français du pétrole, fax. +33147527078 , or revueogst@ifp.fr. 